SMOKING EXPOSURE IN MALE WITH ACUTE MYOCARDIAL INFARCTION UNDERGOING PRIMARY ANGIOPLASTY IS RELATED WITH 1 YEAR PROGNOSIS

doi:10.1136/hrt.2010.208967.531

Ma Qin, Yan Hongbing. Beijing Anzhen Hospital

Objective To evaluate relation between smoking in 1 year events in cardiac death in patients with ST-elevation myocardial infarction (STEMI) undergoing primary percutaneous coronary intervention (PCI).

Methods 624 consecutive patients (506 male, 118 females) with STEMI undergoing primary PCI were enrolled, including 319 smoking female (63.0\%), 187 nonsmoking (37.0\%). To observe the relation between smoking and 1 year cardiac death in male.

Result Male have 25 cases cardiac death (4.9\%), including 11 deaths in administration (2.1\%), smoking 23 cases (4.5\%), nonsmoking 2 cases $(0.39 \%) \mathrm{p}=0.001$. Multivariate liner regression analysis showed that active smoking is associated with SYNTAX score in coronary artery lesions $(p=0.019)$. Logistic regression analysis showed a significant relationship between active smoking and 1 year cardiac death (OR, 3.472; 95\% CI 3.035 to 3.762 ; $p=0.021$ ).

Conclusion Active smoking is related with 1 year cardiac death. Male active smoking is not only the independent risk factor with cardiac death, but also the risk of adverse prognosis after intervention.

\section{e0532 WHY DID WE FAIL OR BE UNABLE TO PREDICT THE INFARCT-RELATED ARTERY BY ECG}

doi:10.1136/hrt.2010.208967.532

Zhang Xiaojiang, Yan Hongbing. Beijing Anzhen Hospital

Objective The infarct-related artery (IRA) can be predicted by ECG, but there are little concern about why the IRA could not be predicted by ECG. In the present study, we attempted to understand why did we fail or be unable to predict the IRA by ECG based on the comparison between ECG records and coronary angiographic findings.

Methods All 18-lead ECG records were compared with their angiographic findings for analysis from 1024 consecutive patients with ST elevation myocardial infarction (STEMI) between October 2004 and July 2009. More than two continous 18-lead ECG records were performed within $12 \mathrm{~h}$ of the onset of symptoms in all patients. Patients with previous myocardial infarction, previous coronary artery bypass surgery, previous pacemaker implant surgery or ECG evidence of left bundle branch block and time from onset to the period of the angiography more than $12 \mathrm{~h}$ were excluded from this study.

Results Of all 1024 patients enrolled, the IRA were failed to be predicted by ECG in 170 patients: failure prediction in 96 patients and unable evaluation in other 74 patients. Of these 170 patients, IRA was left circumflex coronary artery in $76(44.7 \%)$ patients, right coronary artery in 66 (38.8\%) patients, left anterior descending branch in $20(11.8 \%)$ patients, ramus medianus branch in 7 (4.1\%) patients, and left main in $1(0.6 \%)$ patient. Double-vessel and triplevessel diseases were recorded in 27 (16\%) patients and 47 (28\%) patients respectively. Early repolarisation syndrome occurred in 8 $(5 \%)$ patients, and dextrocardia1 patient $(0.6 \%)$. Angiographic study showed acute occlusion of a small branch in $6(3.6 \%)$ patients.

Conclusion Coronary collateral vessel can mislead judgements of the IRA. When the IRA cannot be determined by ECG, left circumflex coronary artery is most likely to be the culprit vessel. Occasionally, early repolarisation syndrome and anatomic variation of the coronary artery or heart and a small branch occlusion could be causes of misjudgements.

\section{e0533 THE EFFECT OF OCA IN LEFT MAIN LESION}

doi:10.1136/hrt.2010.208967.533

Qin Qing, Qian Juying, Fan Bin, Wang Qibin, Ge Lei, Ge Junbo. Zhongshan Hospital

Objective Using Intravascular ultrasound (IVUS) as a standard to evaluate the ability of Quantitative Coronary Angiography (OCA) in judging the lumen diameter before and after percutaneous coronary intervention (PCI) in left main lesion, in choosing stent before $\mathrm{PCI}$ and also in evaluating the effect of PCI.

Method A retrospective study was conducted including left main lesion completing IVUS study which either has a diameter stenosis exceeding $50 \%$ by visual measure or a dissection confirmed by coronary angiography (CAG) in the past 4 yrs. CAAS was used in analysing CAG data and the extent of left main lesion was measured in a position with best exposure of left main coronary artery (cranial or caudal). Echoplaque was used to analyse IVUS data.

Result From June 2004 to December 2008, sixty-four patients met the inclusion criteria including 49 males $(76 \%)$ with an average age of $61.2 \pm 11.2$ years old. In all the lesions underwent IVUS study, there are 18 ostial lesions, 8 trunk lesions, 17 distal lesions without bifurcation involvement, 7 distal lesions with either LAD or LCX involved, 7 distal lesions with both LAD and LCX involved, 11 diffuse lesions and 2 cases of left main dissection. Of them, 6 patients had two sites of lesions. Fifteen patients were not able to complete QCA study before the PCI procedure and the relevant lesions including one case of ostial lesion, 6 cases of distal lesion with bifurcation involved and 8 cases of diffuse lesion. Before PCI, the average minimum lumen diameter analysed by QCA and IVUS was $1.50 \pm 0.73 \mathrm{~mm}, 1.82 \pm 0.59 \mathrm{~mm}$ respectively; reference lumen diameter was $4.02 \pm 0.71 \mathrm{~mm}, 3.74 \pm 0.63 \mathrm{~mm}$ separately. After PCI, the average minimum in-stent lumen diameter by QCA and IVUS was $3.75 \pm 0.53 \mathrm{~mm}, 3.25 \pm 0.66 \mathrm{~mm}$; and the reference lumen diameter was $4.06 \pm 0.49 \mathrm{~mm}, 3.77 \pm 0.60 \mathrm{~mm}$ respectively. Relative analysis showed the correlation coefficient of minimum lumen diameter and reference lumen diameter before PCI, minimum instent lumen diameter and reference lumen diameter after PCI was $0.72(p<0.001), 0.47(p<0.05), 0.73(p<0.001), 0.79(p<0.001)$, respectively.

Conclusion QCA has a high correlation with IVUS measurement and can be used in guiding and evaluating PCI procedure of left main disease. However, its use is limited in diffuse lesion and distal lesion involving bifurcation of left main coronary artery and a high quality CAG image is required.

\section{e0534 THE ASSESSMENT OF SIGNIFICANCE OF INTERMEDIATE CORONARY STENOSIS WITH INTRAVASCULAR ULTRASOUND}

doi:10.1136/hrt.2010.208967.534

Fang Zhe, Zhou Yujie, Shi Dongmei, Zhao Yingxin, Liu Yuyang, Li Yanfang. Department Of Cardiology, Beijing Anzhen Hospital, Capital Medical University, Beijing, China

Objective To compare the detection of stenosis by angiography and identified angiographic predictors of severe luminal stenosis on intravascular ultrasound (IVUS) in patients with intermediate coronary stenosis.

Methods 450 patients with coronary intermediate stenosis by quantitative coronary angiography (OCA) and underwent IVUS assessment of the lesions artery. The results from IVUS and QCA were compared using unpaired t-test. Multiple regression analysis was performed to identify parameters that could predict the presence of severe stenosis on IVUS.

Results A total of 780 stenotic coronary lesions were classified as intermediate by OCA. There was a right correlation between IVUS 